\title{
White matter brain aging In Relationship to Schizophrenia and Its Cognitive Deficit
}

4 Jingtao Wang ${ }^{\mathrm{a}, \mathrm{b}}$, Peter Kochunov ${ }^{\mathrm{b}}$, Hemalatha Sampath ${ }^{\mathrm{b}}$, Kathryn S. Hatch ${ }^{\mathrm{b}}$, Meghann C. Ryan ${ }^{\mathrm{b}}$, Fuzhong 5 Xue $^{\mathrm{a}}$, Jahanshad Neda ${ }^{\mathrm{c}}$, Thompson Paul ${ }^{\mathrm{c}}$, Britta Hahn ${ }^{\mathrm{b}}$, James Gold ${ }^{\mathrm{b}}$, James Waltz ${ }^{\mathrm{b}}$, L. Elliot Hong ${ }^{\mathrm{b}}$, Shuo 6 Chen $^{\mathrm{b}, \mathrm{d}^{*}}$

$8{ }^{a}$ Department of Biostatistics, School of Public Health, Cheeloo College of Medicine, Shandong 9 University

${ }^{\mathrm{b}}$ Maryland Psychiatric Research Center, Department of Psychiatry, University of Maryland School of Medicine, Baltimore, MD, USA

${ }^{\mathrm{c}}$ Imaging Genetics Center, Stevens Institute for Neuroimaging \& Informatics, Keck School of Medicine of USC, Marina del Rey, CA, USA

${ }^{\mathrm{d}}$ Division of Biostatistics and Bioinformatics, Department of Public Health and Epidemiology, University of Maryland School of Medicine, Baltimore, MD, USA

*Corresponding author at: University of Maryland School of Medicine, MPRC building, Room 2-1, 55 Wade Ave, Catonsville, MD 21228, USA

Email address: shuochen@ som.umaryland.edu (S.Chen) 


\section{Abstract}

We hypothesized that cerebral white matter deficits in schizophrenia (SZ) are driven in part by

3 accelerated white matter aging and are associated with cognitive deficits. We used machine learning model

4 to predict individual age from diffusion tensor imaging features and calculated the delta age ( $\Delta$ age $)$ as the

5 difference between predicted and chronological age. Through this approach, we translated multivariate

6 white matter imaging features into an age-scaled metric and used it to test the temporal trends of accelerated

7 aging-related white matter deficit in $\mathrm{SZ}$ and its association with the cognition. Followed feature selection,

8 a machine learning model was trained with fractional anisotropy values in 34 of 43 tracts on a training set

9 consisted of 107 healthy controls (HC). The brain age of $166 \mathrm{SZs}$ and $107 \mathrm{HCs}$ in the testing set were

10 calculated using this model. Then, we examined the SZ-HC group effect on $\Delta$ age and whether this effect

11 was moderated by chronological age using the regression spline model. The results showed that $\triangle$ age was

12 significantly elevated in the age $>30$ group in patients $(p<0.001)$ but not in age $\leqslant 30$ group $(p=0.364)$.

$13 \triangle$ age in patients was significantly and negatively associated with both working memory $(\beta=-0.176, p=$

$140.007)$ and processing speed $(\beta=-0.519, p=0.035)$ while adjusting sex and chronological age. Overall,

15 these findings indicate that the $\Delta$ age is elevated in SZs and become significantly from middle life stage; the 16 increase of $\Delta$ age in SZs is associated with the decline neurocognitive performance.

19 Keywords: BrainAGE; Prediction; Schizophrenia; Diffusion tensor imaging; White matter; Cognitive

20 performance 


\section{Introduction}

Patients with schizophrenia (SZ) are at risk for elevated aging-related functional and neurological decline, termed accelerated aging (Kirkpatrick et al., 2008; Ito and Barnes, 2009; Jeste et al., 2011; Kochunov et al., 2013b, 2014, 2016b). They are at significantly higher risks (1.5 to 5 times) for developing cognitive deficits and dementia before age 70 (Cai and Huang, 2018; Chen et al., 2015; Diniz et al., 2017; Ribe et al., 2015). Previous studies demonstrated that the integrity of the cerebral white matter as measured by fractional anisotropy (FA) in diffusion weighted imaging (DTI), declined in SZ patients at nearly twice the aging rate of normal controls (Mori et al., 2007; Friedman et al., 2008; Kochunov et al., 2013a, 2013b; Wright et al., 2014). An analysis of life-long trajectory of white matter integrity in 600 schizophrenia patients suggested that the peak of white matter integrity occurs earlier in SZ than healthy controls and the accelerated decline of the associative white matter tracts becomes evident in the $4^{\text {th }}$ decade of life and its slope shows a non-linear increase with age (Cetin-Karayumak et al., 2019).

The strong aging related sensitivity of white matter measures such as FA (Kochunov et al., 2016a) can also be used to predict "brain age" for individual subjects using neuroimaging data. The difference between brain age and chronological age can then be used as a phenotype to evaluate evidence for accelerated or slower aging in an individual (Cole and Franke, 2017; Franke et al., 2012; Smith et al., 2019; Wang et al., 2019). The "brain age" analysis can be performed using machine learning and/or regression models that are trained to draw association between regional brain measures and chronological age. The delta age ( $\Delta$ age), the difference between brain age and chronological age, is expected to be null on average in a group of individuals undergoing the normal brain aging process. It translates multivariate imaging features into an age-scaled metric that can be used as an index to depict imaging-based brain structural changes during aging. The $\Delta$ age may be increased by the atypical brain aging caused by physical and brain diseases (Franke et al., 2013, 2012) such as dementia (Wang et al., 2019), Alzheimer's disease (Gaser et al., 2013), schizophrenia (Koutsouleris et al., 2014; Nenadić et al., 2017), or epilepsy (Holmes et al., 2012). 
Patients with schizophrenia (SZ) have a significantly higher risk (2-4 times) of experiencing an

2 unfavorable aging trajectory that may increase their risk for cognitive decline and dementia (Ribe et al.,

3 2015). The increased risk can be in part explained by the fact that the white matter integrity reaches its peak

4 earlier in life for SZ than healthy controls (Cetin-Karayumak et al., 2019). Neuropathology of accelerated

5 brain aging likely contributes to the severity of the cognitive deficits in patients that form the core of

6 socioeconomic impairments in this illness (Kochunov et al., 2016b; Kelly et al., 2018; Kochunov et al.,

7 2017). Previously, we have shown that white matter deficits are associated with cognitive deficits and

8 treatment difficulty in SZ (Kochunov et al., 2019, 2017, 2016b) and have hypothesized that accelerated

9 white matter aging in SZ leads to development of cognitive deficits and disorder-specific deficit patterns

10 (Kochunov and Hong, 2014).

Built on the previous research, we used machine learning to calculate white matter $\Delta$ age and further

12 tested two hypotheses that (1) white matter $\Delta$ age is elevated in patients with schizophrenia and the SZ-

13 related elevation in $\Delta$ age is moderated by chronological age (i.e., white matter $\Delta$ age in $\mathrm{SZ}$ across the

14 lifespan); and (2) the white matter $\Delta$ age is associated with the core cognitive deficits in schizophrenia while

15 adjusting chronological age and other covariates.

\section{2. Materials and methods}

\subsection{Participants}

All the patients were recruited in Maryland Psychiatric Research Center, University of Maryland

20 School of Medicine and neighboring outpatient clinics. Healthy controls were recruited via advertising, e.g.,

21 flyers, social media and word of mouth. Imaging data were available for $\mathrm{N}=166$ individuals with diagnosis

22 of schizophrenia spectrum disorders (117 Male / 49 Female, average age $=36.232)$ and 214 HC (140 Male

23 / 74 Female, average age $=38.016)$ without current Axis I psychiatric illnesses $($ Table 1$)$. Among all SZs,

2411 subjects were diagnosed as schizoaffective disorder while others were diagnosed as schizophrenia. 
1 Diagnosis of schizophrenia spectrum disorders was conducted according to Diagnostic and Statistical

2 Manual of Mental Disorders-IV (DSM-IV) criteria through a best estimate approach combining information

3 from a Structured Clinical Interview for DSM-IV (SCID) with a review of medical records. The exclusion

4 criteria included diagnosis with hypertension, hyperlipidemia, type 2 diabetes, heart disorders, major

5 neurologic event such as stroke or transient ischemic attack, and recent substance use disorder (except

6 tobacco and marijuana use). It was applied for all participants based on self-reported questionnaires. All

7 participants gave written informed consent approved by the University of Maryland Baltimore institutional

8 review board.

To evaluate the prediction performance of the machine learning model, two parts were extracted from

HC frequency-matching the distribution of SZ subjects' age and gender. One part consisted of $107 \mathrm{HC}$ (70

11 Male / 37 Female, average age $=37.822$ ) was used to fit the machine learning model. The other part

12 consisted of $107 \mathrm{HC}$ (70 Male / 37 Female, average age = 38.210) constituted the testing set with SZ

13 subjects together. Additional clinical and epidemiologic information is provided in Table 1.

\subsection{Image Acquisition and Processing}

Magnetic resonance images were acquired through studies using magnetic resonance imaging 3T Siemens scanner. Imaging data was collected using a Siemens 3T TRIO MRI (Erlangen, Germany), running an upgraded VB17 software and a 32-channel RF head coil. DTI data was collected using a spin-echo, EPI sequence with a spatial resolution of $1.7 \times 1.7 \times 3.0 \mathrm{~mm}$. The sequence parameters were: $\mathrm{TE} / \mathrm{TR}=87 / 8000$ $\mathrm{ms}, \mathrm{FOV}=200 \mathrm{~mm}$, axial slice orientation with 50 slices and no gaps, 64 isotropically distributed diffusion weighted directions, two diffusion weighting values $(b=0$ and $700 \mathrm{~s} / \mathrm{mm} 2)$ and five $b=0$ images. Subjects'

21 head movement was minimized with restraining padding. The DTI data were processed using the ENIGMA

22 DTI analysis pipeline (https://www.nitrc.org/projects/enigma_dti) (Jahanshad et al., 2013). All data

23 included in the analysis passed the ENIGMADTI quality assurance or quality control procedures. Regional

24 white matter FA measurements were generated for 43 tracts (Supplementary methods). Among them, the 
1 per-tract mean values were found by calculating the mean values along tract regions of interest per

2 hemisphere except for commissural tracts.

\subsection{Data Analysis and Statistical Modeling}

\subsubsection{Machine learning model for white matter brain age calculation}

A machine learning (ML) i.e. supervised learning computational model is first used to calculate 'white matter brain age' of each participant using only DTI features. This task can be implemented by training an optimal machine learning model in community control participants (Cole and Franke, 2017; Liem et al., 2017; Shahab et al., 2019), locking the model, and applying the model to patients and controls in the testing sample. The training of the ML model is often based on solely the controls (Franke et al., 2012; Khundrakpam et al., 2015), same as we have done here $(\mathrm{N}=107)$. In the training stage, chronological age was considered as the continuous outcomes/labels (Y) and white matter FA values as the input features (X).

To achieve the best prediction performance, several machine learning model candidates including Random forest regression (Breiman, 2001; Geurts et al., 2006), Gradient boosting regression(Friedman, 2002, 2001), LASSO (Least absolute shrinkage and selection operator) (Friedman et al., 2010) and others were utilized and compared. The feature selection, parameter tuning of each model and model comparison were conducted using 5-fold cross-validation (CV) within the training data set. The criteria of prediction performance were the coefficients of determination $\left(R^{2}\right)$ between the chronological and brain age (which is equivalent to the mean absolute error (MAE) criteria) (Fig 1A). The best performing model in the CV then was locked and applied to the testing sample, thus the testing sample results were not influenced by the training data. Note that the demographic variables are balanced between the training and testing data sets, and between testing $\mathrm{HC}$ and SZ groups.

Next, the locked machine learning model was applied to the testing data set which includes both SZ $(\mathrm{N}=166)$ and $\mathrm{HC}(\mathrm{N}=107)$. The model was used to calculate the 'brain age' based on the input brain 
1 imaging features (Fig 1B). The brain age delta ( $\Delta$ age) was then calculated. A positive value would suggest

2 an accelerated white matter aging effect.

A

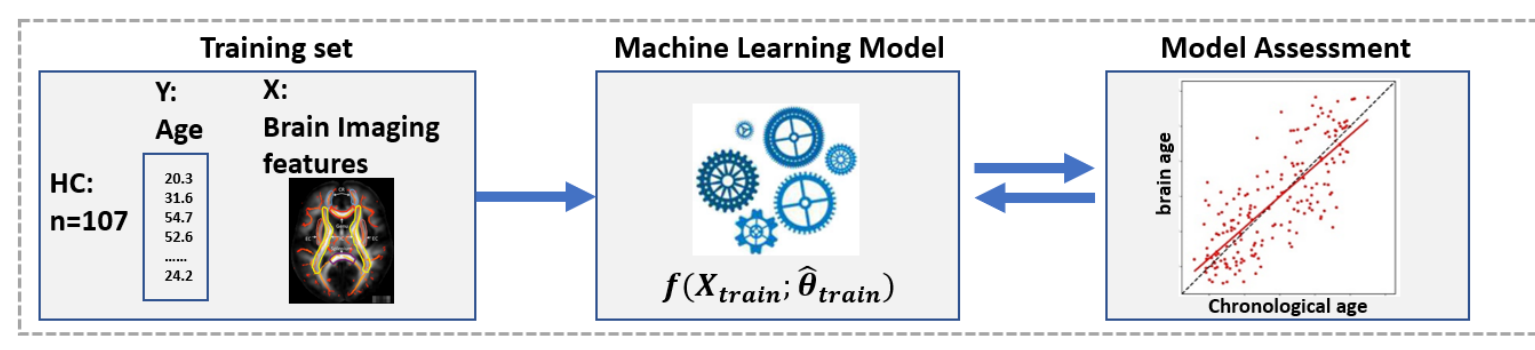

B

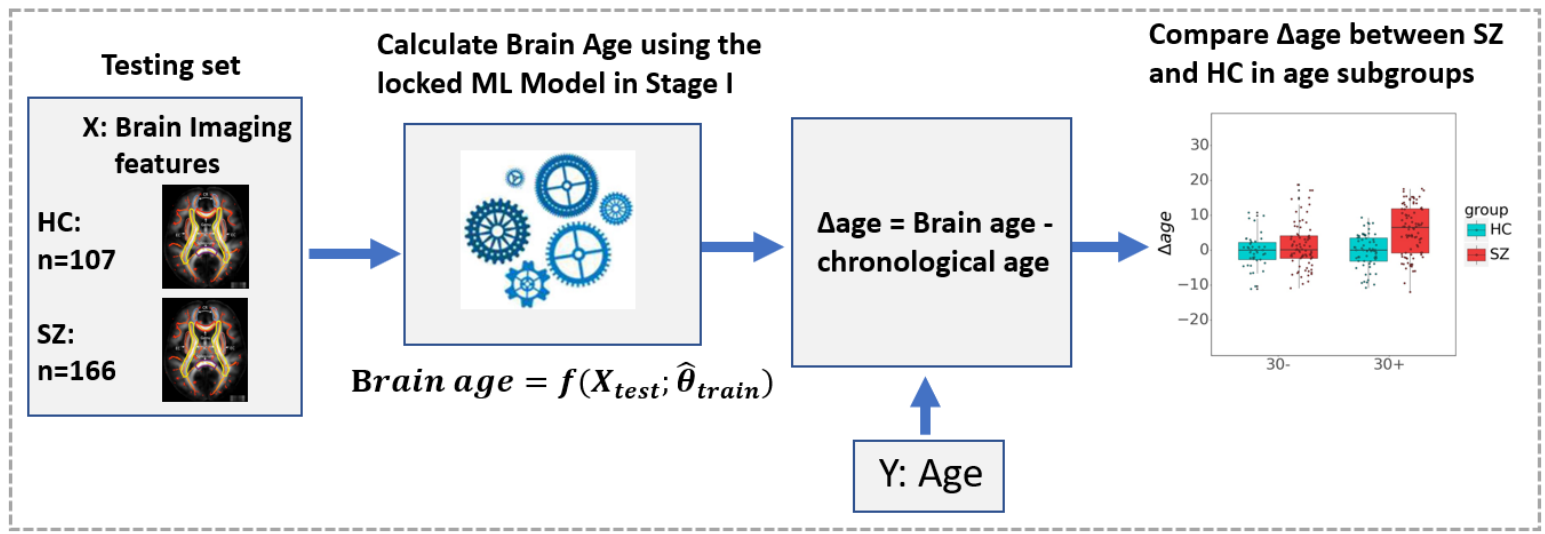

Fig. 1. Flowchart showing the process of study design. (A) Training: Machine learning model estimation in the testing data set in two age groups.

\subsubsection{Statistical analysis of $\Delta$ age}

We first tested whether white matter $\Delta$ age is associated with SZ, and whether the diagnosis group

13 effect is moderated by chronological age. Since the moderation effect of chronological age is not linear, we

14 applied a comprehensive and data-driven approach to objectively determine the disease effect on $\Delta$ age 
1 across the lifespan. Specifically, we used spline models including linear splines and natural cubic splines

2 as basis functions to assess the moderation effect of the chronological age (Wood, 2011). Then, we

3 performed model selection and chose the optimal location and number of knots and spline basis function

4 based on the likelihood criteria (e.g., BIC). The final model can provide statistical inference to evaluate the

5 effect of SZ on $\triangle$ age across the lifespan. We then tested the difference of $\Delta$ age between SZ and $\mathrm{HC}$ in each

6 age subgroup using two sample tests after correction for multiple comparisons; note that age and sex were

7 frequency-matched between SZ and HC.

8 We also examined the association between $\Delta$ age and cognitive deficits in schizophrenia patients. The

9 Wechsler Abbreviated Scale of Intelligence digit-sequencing and digit-symbol-coding subscales were used

10 to assessed working memory and processing speed, respectively (Wechsler, 1999); these tasks were selected

11 as they are among the most robust tasks separating those with schizophrenia spectrum disorders vs. controls

12 in meta-analysis across all cognitive domains (Dickinson et al., 2007). These metrics were regressed against

$13 \Delta$ age independently using the general linear model (GLM). Since $\Delta$ age was hypothesized to be elevated in

14 SZ but was expected to be null on average in $\mathrm{HC}$ along lifespan, we assessed the impact of $\Delta$ age on 15 cognitive deficits in both SZ and HC group (Wang et al., 2007). Chronological age and sex were adjusted 16 as covariates. In addition, to assess the robustness of results, we repeated the statistical analysis by 17 excluding the schizoaffective disorder patients.

\section{Results}

\subsection{Participants characteristics}

22 gender between the three parts of data set ( $p=0.439, p=0.579$, respectively). 


\begin{tabular}{|c|c|c|c|c|c|}
\hline \multirow{2}{*}{ Variable } & \multirow{2}{*}{ Level } & Training data set & \multicolumn{3}{|c|}{ Testing data set } \\
\hline & & $\mathrm{HC}(\mathrm{N}=107)$ & $\mathrm{SZ}(\mathrm{N}=166)$ & $\mathrm{HC}(\mathrm{N}=107)$ & $p$ \\
\hline Age & mean $\pm \mathrm{SD}$ & $37.822 \pm 13.603$ & $36.232 \pm 13.195$ & $38.210 \pm 14.243$ & 0.439 \\
\hline Age & median [range] & $\begin{array}{l}33.974[19.129, \\
63.817]\end{array}$ & $\begin{array}{l}31.704[18.092, \\
63.444]\end{array}$ & $\begin{array}{l}35.283[18.401, \\
63.499]\end{array}$ & 0.495 \\
\hline Gender & Female & $37(34.579)$ & $49(29.518)$ & 37 (34.579) & 0.579 \\
\hline & Male & $70(65.421)$ & $117(70.482)$ & $70(65.421)$ & \\
\hline Age of Onset & mean $\pm \mathrm{SD}$ & - & $23.088 \pm 6.007$ & - & - \\
\hline Duration & mean $\pm \mathrm{SD}$ & - & $10.473 \pm 11.515$ & - & \\
\hline $\mathrm{CPZ}$ & mean $\pm \mathrm{SD}$ & - & $398.581 \pm 405.868$ & - & \\
\hline
\end{tabular}

\section{2}

\section{3.2. Machine learning model selection on the training data set}

4 The random forest regression model achieved the best performance in predicting the age in the test-retest

5 trials in HC participants. The features selection in random forest model was conducted using the recursive

6 feature elimination (Breiman, 2001; Geurts et al., 2006). Among 43 tracts, FA values of 34 tracts were

7 selected (Supplementary methods). The parameter tuning of random forest was conducted using 5-fold CV

8 with the $R^{2}$ as the criteria of prediction performance. The random forest model predicted chronological age

9 with an $R^{2}$ of 0.916 (MAE $=4.640$ years, $p<0.001$ ) and achieved good performance when be applied to

$10 \mathrm{HC}\left(R^{2}=0.895, \mathrm{MAE}=3.649\right.$ years, $\left.p<0.001\right)$ and $\mathrm{SZ}\left(R^{2}=0.814\right.$, MAE $=6.469$ years, $\left.p<0.001\right)$ in the

11 testing dataset (Supplementary Fig. S1). The performance of other models was inferior and presented in the

12 supplementary Table S1. 


\section{3.3. Accelerated aging in patients}

2 The correlation between chronological age and $\Delta$ age were significantly in SZ $\left(R^{2}=0.048, p=0.005\right)$ but

3 not significantly in $\mathrm{HC}\left(R^{2}=0.010, p=0.296\right)$ in the testing dataset (Supplementary Fig. S2). The effect of

4 SZ by age interaction on $\Delta$ age was positive and significant $(\beta=0.134, p=0.007)$, which indicated the

5 effect of group on $\Delta$ age is moderated by chronological age. Furthermore, we examined the SZ-HC group

6 effect on $\Delta$ age, and the nonlinear moderation effect of chronological age on the group effect using the

7 regression spline model. Based on the likelihood criteria, the final model which fitted the group effect on

$8 \Delta$ age across the lifespan optimally is a linear spline model with one knot at age $=30$. Based on this age

9 knot, the chronological age was divided into two subgroups ( $\leqslant 30$ years old and $>30$ years old). The $\Delta$ age

10 was then compared between SZs and HCs for these two age groups. The $\triangle$ age of the SZs was not

11 significantly higher than that of HCs in $\leqslant 30$ age group (mean difference (95\% CI): $1.479(-0.586,3.543)$,

$12 p=0.364)$ but was significantly higher in $>30$ age group $(5.903(3.989,7.817), p<0.001)$ (Fig. 2). The

13 demographic characteristics of testing set in two age groups are presented in Table 2. 


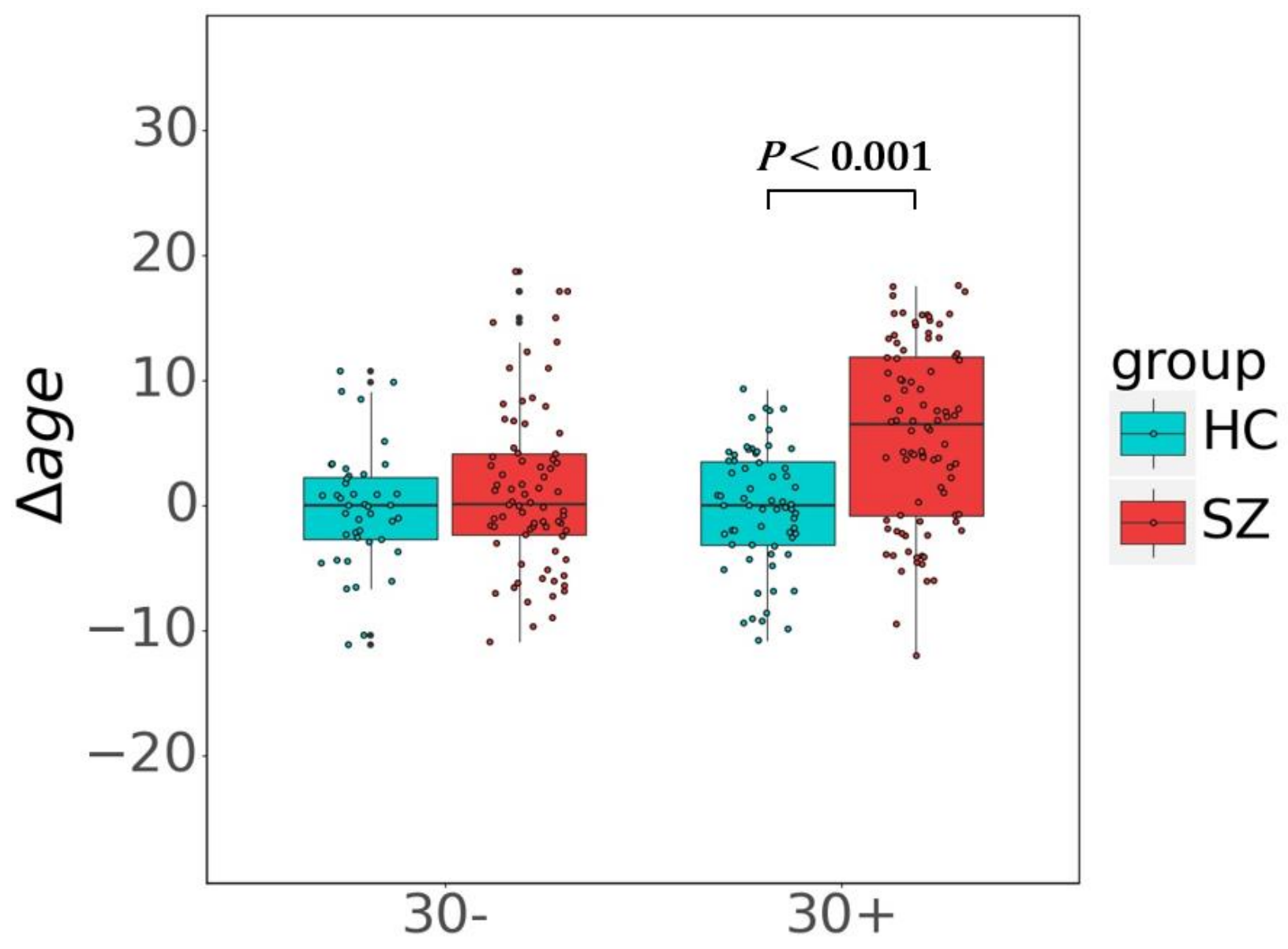

2 Fig. 2. Box plots demonstrate the differences of $\Delta$ age between SZ and HC in the testing data set for two

3 age groups. Significant $\Delta$ age difference between $\mathrm{SZ}$ and $\mathrm{HC}$ emerged at age group 30+. No significant

4 differences were found in the younger age group.

5

6 Table 2. Demographics and clinical characteristics of participants in each age group in testing set

\begin{tabular}{|c|c|c|c|c|c|}
\hline \multirow{2}{*}{ Variable } & \multirow{2}{*}{ Level } & \multicolumn{2}{|c|}{ age $\leq 30$} & \multicolumn{2}{|c|}{ age $>30$} \\
\hline & & $\mathrm{SZ}(\mathrm{N}=76)$ & $\mathrm{HC}(\mathrm{N}=43)$ & $\mathrm{SZ}(\mathrm{N}=90)$ & $\mathrm{HC}(\mathrm{N}=64)$ \\
\hline Age & mean $\pm \mathrm{SD}$ & $24.204 \pm 3.374$ & $23.74 \pm 3.145$ & $46.389 \pm 9.238$ & $47.932 \pm 9.788$ \\
\hline
\end{tabular}




$\begin{array}{lllll}\text { median } & 24.726[18.092, & 23.606[18.401, & 48.168[30.313, & 48.905[30.226, \\ \text { [range }] & 29.610] & 29.788] & 63.444] & 63.499]\end{array}$

$\begin{array}{lllll}\text { Gender } & \text { Female } & 18(23.684) & 15(34.884) & 31(34.444)\end{array}$

$\begin{array}{lll}\text { Male } \quad 58(76.316) & 28(65.116) & 59(65.556)\end{array}$

$\begin{array}{llll}\text { Age of Onset } \quad \text { mean } \pm \text { SD } & 18.536 \pm 3.024 \quad- & 22.913 \pm 8.005\end{array}$

$\begin{array}{llll}\mathrm{CPZ} & \text { mean } \pm \mathrm{SD} & 340.169 \pm 340.916 \quad- & 554.702 \pm 443.104\end{array}$

\section{3.4. Assessing the correlation between $\Delta$ age and cognition}

3 In patient, $\Delta$ age was significantly associated with both working memory $(\beta=-0.176, p=0.007)$ and

4 processing speed $(\beta=-0.519, p=0.035)$ after adjusting sex and chronological age (Table 3$)$. Average daily

5 antipsychotic medications as measured by Chlorpromazine equivalent (CPZ) was not significantly

6 associated with $\Delta$ age in the patients $(\beta=0.002, p=0.179)$. Age of psychosis onset in the patients was also

7 not significantly associated with $\Delta$ age $(\beta=-0.007, p=0.970)$. Finally, duration of the illness in the patients

8 was also not significantly associated with $\Delta$ age $(\beta=0.110, p=0.165)$. In the controls, neither working

9 memory $(\beta=-0.059, p=0.636)$ nor processing speed measures $(\beta=-0.180, p=0.618)$ was significantly

10 associated with $\Delta$ age after adjusting sex and chronological age (Table 3, Fig 3). The results showed that

$11 \Delta$ age and cognitive deficits are correlated only for patients with schizophrenia, but not for healthy controls.

12 We also performed regression analysis using the combined SZ and HC cohort ( $=273)$ with covariates of

13 chronological age, $\Delta$ age, sex, group, chronological age $\times$ group, and $\Delta$ age $\times$ group. The results were

14 similar to the subgroup analysis and summarized in Supplementary table S2. In addition, we repeated the

15 statistical analysis by excluding the schizoaffective disorder patients, and the results remained similar to

16 the main results (supplementary materials). 
bioRxiv preprint doi: https://doi.org/10.1101/2020.10.19.344879; this version posted October 20, 2020. The copyright holder for this preprint (which was not certified by peer review) is the author/funder, who has granted bioRxiv a license to display the preprint in perpetuity. It is made available under aCC-BY-NC-ND 4.0 International license.

Table 3. Associations between two age-scaled metrics and cognition

\begin{tabular}{|c|c|c|c|c|c|}
\hline \multirow{2}{*}{ Group } & \multirow{2}{*}{ Variable } & \multicolumn{2}{|c|}{ Working memory } & \multicolumn{2}{|c|}{ Processing speed } \\
\hline & & $\beta$ & $p$ & $\beta$ & $p$ \\
\hline \multirow[t]{3}{*}{$\mathrm{SZ}$} & Sex & -1.530 & 0.147 & 7.784 & 0.053 \\
\hline & chronological age & -0.067 & 0.053 & -0.350 & $0.009 * *$ \\
\hline & $\Delta$ age & -0.176 & $0.007 * *$ & -0.519 & $0.035^{*}$ \\
\hline \multirow[t]{3}{*}{$\mathrm{HC}$} & Sex & -0.900 & 0.502 & 1.999 & 0.604 \\
\hline & chronological age & -0.020 & 0.636 & -0.674 & $<0.001 * * *$ \\
\hline & $\Delta$ age & -0.059 & 0.636 & -0.180 & 0.618 \\
\hline
\end{tabular}
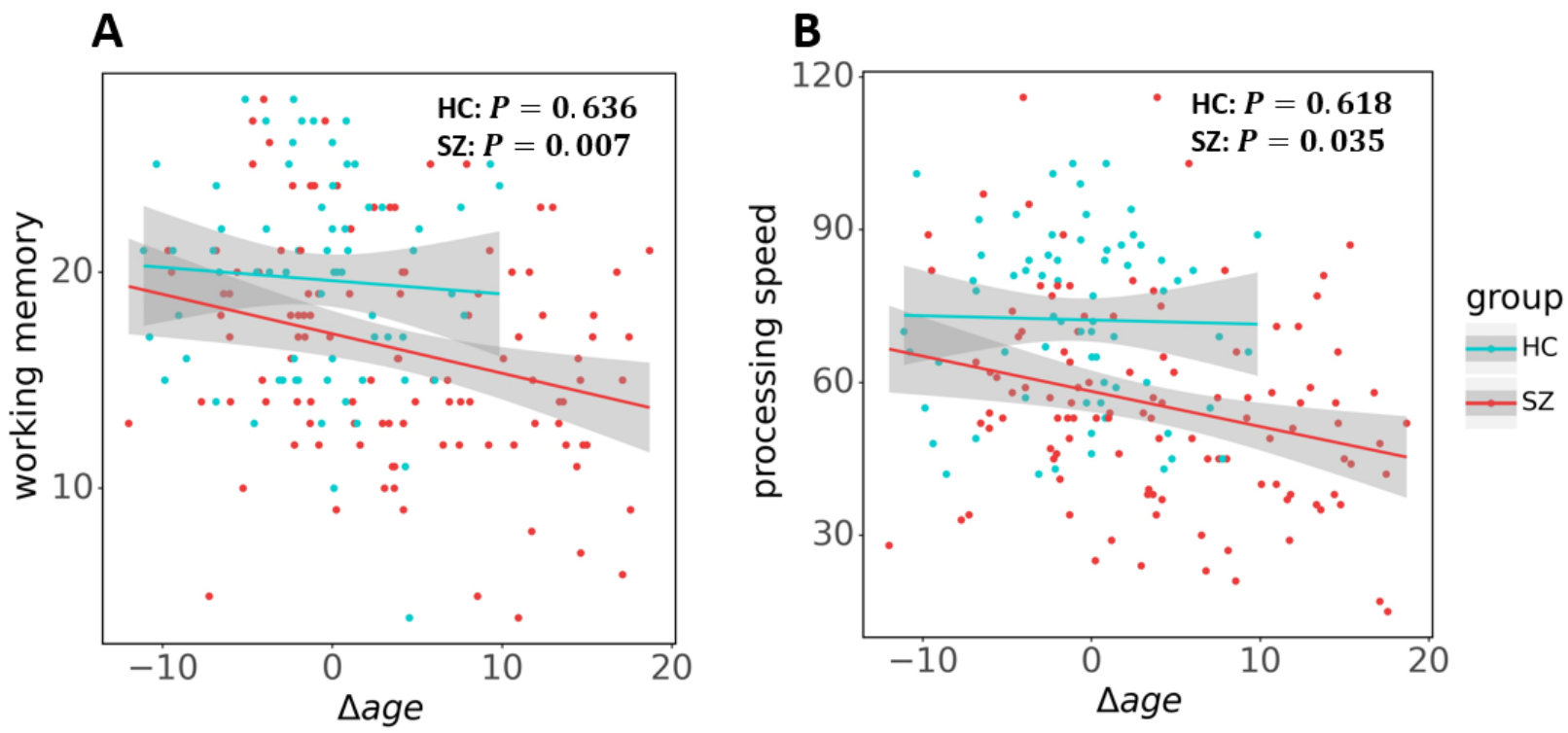

5 Fig. 3. Scatterplots and linear fits illustrating relationships between $\Delta$ age and working memory and 6 processing speed in patients with schizophrenia and healthy controls. 


\section{Discussion}

In the current study, a novel Brain Age analysis approach was applied to perform personalized predictions of individual age based on white matter imaging. The calculated white matter $\Delta$ age, the difference between predicted and chronological ages, was elevated in SZ compared to healthy controls and the difference of $\Delta$ age between SZ and HC was moderated by chronological age. Followed determining the optimal age knot based on the likelihood principle, we assigned participants into two age groups and found that $\Delta$ age was significantly higher in $\mathrm{SZ}$ relative to $\mathrm{HC}$ at age group old than 30 years old but not significantly at age group younger than 30 years old. Furthermore, we also identified that $\Delta$ age in patients was associated with worsened performance on two of the neurocognitive tests most affected in schizophrenia: working memory and processing speed.

Most recently, using machine learning to predict the age of an individual based on neuroimaging has received increased interest. An increasing number of studies tried to construct brain age in this approach based on varieties of neuroimaging data, such as diffusion tensor imaging, cortical thickness or cognitive performance scores, and further investigated whether the brain age of patients with schizophrenia were higher than those of the healthy controls. Three previous studies in SZ used Brain Age approach with structural T1w data and showed that the average $\Delta$ age in patients was significantly higher (2.6 to 8.8 years) compared to healthy controls (Franke et al., 2010; Hajek et al., 2019; Steffener et al., 2016). Koutsouleris and colleagues also showed that patients with schizophrenia had significantly higher average $\Delta$ age than patients with major depressive or borderline personality disorders (Koutsouleris et al., 2014). Hajek et al. reported a significantly higher $\Delta$ age even in the first-episode schizophrenia patients compared with controls. Together, these studies provided strong evidence that participants with schizophrenia experience an accelerated aging compared to HCs, however the timing of these changes remained unclear (Hajek et al., 2019).

This is the first brain age analysis in SZ that was focused on cerebral white matter. We demonstrated that $\Delta$ age was significantly greater in patients than healthy controls thus supporting previous reports of 
1 accelerated white matter aging in this illness (Cetin-Karayumak et al., 2019; Kochunov et al., 2013b).

2 Moreover, we found no significant differences in brain age among young patients and $\Delta$ age only became

3 significant in middle age in SZ. Our age group analysis of $\Delta$ age differences among two subgroups further

4 suggests that accelerated decline in FA may represent the life-long interaction with schizophrenia. The lack

5 of significant differences in $\Delta$ age in ages $\leqslant 30$ is interesting. A study by Cetin- Karayumak and colleagues

6 summarized three possible trajectories reported in previous studies and investigated which white matter

7 trajectory fitted best based on a large cohort. These three possible trajectories included: (1)

8 "neurodevelopmental models in schizophrenia" postulating that schizophrenia is caused by environmental

9 and/or genetic insults that occur during prenatal, perinatal, or early childhood/adolescence, leading to lower

10 and parallel trajectories throughout the lifespan as compared to healthy controls; (2) "maturational model

11 in schizophrenia" suggesting that disturbances during maturation would cause different ascending slopes

12 and a shift in peak while the similar starting and end points of trajectories compared to healthy controls,

13 indicating perturbed myelination; (3) "accelerated aging in schizophrenia" supporting that schizophrenia is

14 marked by similar trajectory during maturation of white matter but steeper descending slopes related to

15 accelerated aging processes, such as myelin breakdown. They found that whole-brain FA showed a

16 monotonic increase until reaching a peak at the age of 33 years in controls, and earlier, at the age of 27

17 years in patients and the confidence interval of FA trajectory in two groups overlapped in this period. FA declined monotonically after reaching peak in both patients and controls, and demonstrated accelerated decline rate in patients compared to controls (Cetin-Karayumak et al., 2019). This finding supported the

21 cerebral white matter FA increases in patients and the accelerated increase in $\Delta$ age of patients after 30 years

22 old in our study.

Previous studies have reported that FA deficits are associated with cognitive deficits in schizophrenia

24 (Epstein et al., 2014; Miyata et al., 2010; Nazeri et al., 2013; Pérez-Iglesias et al., 2010; Roalf et al., 2013;

25 Voineskos et al., 2013). Karlsgodt and colleagues observed that lower FA values were predictive of 
1 negative changes in cognitive status (Karlsgodt et al., 2009). Previously, we have hypothesized that

2 accelerated white matter aging in SZ may leads to development of cognitive deficits (Kochunov and Hong,

3 2014). In the current study, we calculated $\Delta$ age through the novel Brain age analysis approach and tested

4 its association with cognitive deficits, and showed that $\Delta$ age in patients was significantly and negatively

5 correlated with performance on the working memory and processing speed neurocognitive tasks. All

6 correlations were negative, indicating that patients who were predicted to be older than their chronological

7 age had worse neurocognitive performance on average. The neurocognitive deficits in these two domains

8 of schizophrenia are enduring, pervasive, and form the core of the functional disability in patients

9 (Dickinson et al., 2007; Faraone et al., 2000; Keefe et al., 2005, 2004; Knowles et al., 2010). The neurocognitive deficits in these domains have already been linked with reduced white matter integrity in

11 patients, suggesting that integrity of long-distance neuronal fibers is critical to maintaining normal

12 performance of long-distance cortical networks that serve these functions (Kochunov et al., 2017). $\Delta$ age

13 does not measure the underlying neurobiological mechanisms. However, it provides an aggregate measure

14 of the white matter deficit pattern along chronological age. In our recent study, we observed the white

15 matter deficit patterns in SZ are strikingly similar with that in Alzheimer disease and are associated with

16 working memory and processing speed. Moreover, the similarity between the white matter deficit patterns

17 in these two diseases were positively correlated with age (Kochunov et al., 2020). This increasing similarity

18 along age may be reflected by $\Delta$ age and hence cause the association between $\Delta$ age and cognitive

19 performance. The highest correlation was observed with the working memory function. Working memory

20 deficits are among the core cognitive deficits reported in schizophrenia (Dickinson et al., 2007; Knowles et

21 al., 2010). Working memory deficits are also directly linked to white matter in both subjects with

22 schizophrenia and normal controls (Karlsgodt et al., 2009; Nazeri et al., 2013; Kochunov et al., 2017; Zeng

23 et al., 2016; Karlsgodt et al., 2010).

There are several limitations in the current study. First, the cross-sectional design may limit our ability 
age of onset on $\Delta$ age in the patients, although they were not significantly associated with $\Delta$ age in the current analysis. A longitudinal DTI study will be necessary to determine the white matter decline trajectories of normal individuals, which will enable estimation of more precisely the effect of schizophrenia on accelerated white matter aging and provide causal inference. Second, the etiology causing the pattern of accelerated white matter aging described here is unknown and not studied in the current study, although we found that current dose of antipsychotic medications that patients were on did not significantly contribute to their $\Delta$ age. elevated compared with healthy controls in relationship to their chronological age and identified that this abnormality occurred at middle and older age and was not significant in young SZ patients. The white matter $\Delta$ age can be used as a simple, individual-level indicator that reflects accelerated white matter brain aging in SZ based imaging features. The pattern of accelerated brain aging in SZ along the lifespan in our analysis can help to reveal the dynamic disease progression of SZ on brain aging and the associated reduction of neurocognitive performance.

\section{Reference}

Breiman, L., 2001. Random Forests. Mach. Learn. 45, 5-32. https://doi.org/10.1023/A:1010933404324

Cai, L., Huang, J., 2018. Schizophrenia and risk of dementia: a meta-analysis study. Neuropsychiatr. Dis. Treat. 14, 2047-2055. https://doi.org/10.2147/NDT.S172933

Cetin-Karayumak, S., Di Biase, M.A., Chunga, N., Reid, B., Somes, N., Lyall, A.E., Kelly, S., Solgun, B., Pasternak, O., Vangel, M., Pearlson, G., Tamminga, C., Sweeney, J.A., Clementz, B., Schretlen, D., Viher, P.V., Stegmayer, K., Walther, S., Lee, J., Crow, T., James, A., Voineskos, A., Buchanan, R.W., Szeszko, P.R., Malhotra, A.K., Hegde, R., McCarley, R., Keshavan, M., Shenton, M., Rathi, Y., Kubicki, M., 2019. White matter abnormalities across the lifespan of schizophrenia: a harmonized multi-site diffusion MRI study. Mol. Psychiatry. https://doi.org/10.1038/s41380-0190509-y

Chen, M.-H., Li, C.-T., Tsai, C.-F., Lin, W.-C., Chang, W.-H., Chen, T.-J., Pan, T.-L., Su, T.-P., Bai, Y.M., 2015. Risk of subsequent dementia among patients with bipolar disorder or major depression: a nationwide longitudinal study in Taiwan. J. Am. Med. Dir. Assoc. 16, 504-508. https://doi.org/10.1016/j.jamda.2015.01.084 
Cole, J.H., Franke, K., 2017. Predicting Age Using Neuroimaging: Innovative Brain Ageing Biomarkers. Trends Neurosci. 40, 681-690. https://doi.org/10.1016/j.tins.2017.10.001

Dickinson, D., Ramsey, M.E., Gold, J.M., 2007. Overlooking the obvious: a meta-analytic comparison of digit symbol coding tasks and other cognitive measures in schizophrenia. Arch. Gen. Psychiatry 64, 532-542. https://doi.org/10.1001/archpsyc.64.5.532

Diniz, B.S., Teixeira, A.L., Cao, F., Gildengers, A., Soares, J.C., Butters, M.A., Reynolds, C.F., 2017. History of Bipolar Disorder and the Risk of Dementia: A Systematic Review and Meta-Analysis. Am. J. Geriatr. Psychiatry Off. J. Am. Assoc. Geriatr. Psychiatry 25, 357-362. https://doi.org/10.1016/j.jagp.2016.11.014

Epstein, K.A., Cullen, K.R., Mueller, B.A., Robinson, P., Lee, S., Kumra, S., 2014. White Matter Abnormalities and Cognitive Impairment in Early-Onset Schizophrenia-Spectrum Disorders. J. Am. Acad. Child Adolesc. Psychiatry 53, 362-372.e2. https://doi.org/10.1016/j.jaac.2013.12.007

Faraone, S.V., Seidman, L.J., Kremen, W.S., Toomey, R., Pepple, J.R., Tsuang, M.T., 2000. Neuropsychologic functioning among the nonpsychotic relatives of schizophrenic patients: the effect of genetic loading. Biol. Psychiatry 48, 120-126. https://doi.org/10.1016/s00063223(99)00263-2

Franke, K., Gaser, C., Manor, B., Novak, V., 2013. Advanced BrainAGE in older adults with type 2 diabetes mellitus. Front. Aging Neurosci. 5. https://doi.org/10.3389/fnagi.2013.00090

Franke, K., Luders, E., May, A., Wilke, M., Gaser, C., 2012. Brain maturation: Predicting individual BrainAGE in children and adolescents using structural MRI. NeuroImage 63, 1305-1312. https://doi.org/10.1016/j.neuroimage.2012.08.001

Franke, K., Ziegler, G., Klöppel, S., Gaser, C., 2010. Estimating the age of healthy subjects from T1weighted MRI scans using kernel methods: Exploring the influence of various parameters. NeuroImage 50, 883-892. https://doi.org/10.1016/j.neuroimage.2010.01.005

Friedman, J.H., 2002. Stochastic gradient boosting. Comput. Stat. Data Anal. 38, 367-378. https://doi.org/10.1016/S0167-9473(01)00065-2

Friedman, J.H., 2001. Greedy function approximation: A gradient boosting machine. Ann. Stat. 29, 11891232. https://doi.org/10.1214/aos/1013203451

Friedman, J.H., Hastie, T., Tibshirani, R., 2010. Regularization Paths for Generalized Linear Models via Coordinate Descent. J. Stat. Softw. 33, 1-22. https://doi.org/10.18637/jss.v033.i01

Friedman, J.I., Tang, C., Carpenter, D., Buchsbaum, M., Schmeidler, J., Flanagan, L., Golembo, S., Kanellopoulou, I., Ng, J., Hof, P.R., Harvey, P.D., Tsopelas, N.D., Stewart, D., Davis, K.L., 2008. Diffusion tensor imaging findings in first-episode and chronic schizophrenia patients. Am. J. Psychiatry 165, 1024-1032. https://doi.org/10.1176/appi.ajp.2008.07101640

Gaser, C., Franke, K., Klöppel, S., Koutsouleris, N., Sauer, H., Initiative, A.D.N., 2013. BrainAGE in Mild Cognitive Impaired Patients: Predicting the Conversion to Alzheimer's Disease. PLOS ONE 8, e67346. https://doi.org/10.1371/journal.pone.0067346

Geurts, P., Ernst, D., Wehenkel, L., 2006. Extremely randomized trees. Mach. Learn. 63, 3-42. https://doi.org/10.1007/s10994-006-6226-1

Hajek, T., Franke, K., Kolenic, M., Capkova, J., Matejka, M., Propper, L., Uher, R., Stopkova, P., Novak, T., Paus, T., Kopecek, M., Spaniel, F., Alda, M., 2019. Brain Age in Early Stages of Bipolar Disorders or Schizophrenia. Schizophr. Bull. 45, 190-198. https://doi.org/10.1093/schbul/sbx172

Holmes, G.L., Milh, M.D.M., Dulac, O., 2012. Chapter 7 - Maturation of the human brain and epilepsy, in: Stefan, H., Theodore, W.H. (Eds.), Handbook of Clinical Neurology, Epilepsy. Elsevier, pp. 135143. https://doi.org/10.1016/B978-0-444-52898-8.00007-0

Ito, K., Barnes, P.J., 2009. COPD as a disease of accelerated lung aging. Chest 135, 173-180. https://doi.org/10.1378/chest.08-1419

Jahanshad, N., Kochunov, P.V., Sprooten, E., Mandl, R.C., Nichols, T.E., Almasy, L., Blangero, J., Brouwer, R.M., Curran, J.E., de Zubicaray, G.I., Duggirala, R., Fox, P.T., Hong, L.E., Landman, B.A., Martin, N.G., McMahon, K.L., Medland, S.E., Mitchell, B.D., Olvera, R.L., Peterson, C.P., Starr, J.M., Sussmann, J.E., Toga, A.W., Wardlaw, J.M., Wright, M.J., Hulshoff Pol, H.E., Bastin, 
M.E., McIntosh, A.M., Deary, I.J., Thompson, P.M., Glahn, D.C., 2013. Multi-site genetic analysis of diffusion images and voxelwise heritability analysis: A pilot project of the ENIGMA-DTI working group. NeuroImage 81, 455-469. https://doi.org/10.1016/j.neuroimage.2013.04.061

Jeste, D.V., Wolkowitz, O.M., Palmer, B.W., 2011. Divergent trajectories of physical, cognitive, and psychosocial aging in schizophrenia. Schizophr. Bull. 37, 451-455. https://doi.org/10.1093/schbul/sbr026

Karlsgodt, K.H., Kochunov, P., Winkler, A.M., Laird, A.R., Almasy, L., Duggirala, R., Olvera, R.L., Fox, P.T., Blangero, J., Glahn, D.C., 2010. A Multimodal Assessment of the Genetic Control over Working Memory. J. Neurosci. 30, 8197-8202. https://doi.org/10.1523/JNEUROSCI.035910.2010

Karlsgodt, K.H., Niendam, T.A., Bearden, C.E., Cannon, T.D., 2009. White matter integrity and prediction of social and role functioning in subjects at ultra-high risk for psychosis. Biol. Psychiatry 66, 562569. https://doi.org/10.1016/j.biopsych.2009.03.013

Keefe, R.S.E., Eesley, C.E., Poe, M.P., 2005. Defining a cognitive function decrement in schizophrenia. Biol. Psychiatry 57, 688-691. https://doi.org/10.1016/j.biopsych.2005.01.003

Keefe, R.S.E., Goldberg, T.E., Harvey, P.D., Gold, J.M., Poe, M.P., Coughenour, L., 2004. The Brief Assessment of Cognition in Schizophrenia: reliability, sensitivity, and comparison with a standard neurocognitive battery. Schizophr. Res. 68, 283-297. https://doi.org/10.1016/j.schres.2003.09.011

Kelly, S., Jahanshad, N., Zalesky, A., Kochunov, P., Agartz, I., Alloza, C., Andreassen, O.A., Arango, C., Banaj, N., Bouix, S., Bousman, C.A., Brouwer, R.M., Bruggemann, J., Bustillo, J., Cahn, W., Calhoun, V., Cannon, D., Carr, V., Catts, S., Chen, J., Chen, J.-X., Chen, X., Chiapponi, C., Cho, K.K., Ciullo, V., Corvin, A.S., Crespo-Facorro, B., Cropley, V., De Rossi, P., Diaz-Caneja, C.M., Dickie, E.W., Ehrlich, S., Fan, F.-M., Faskowitz, J., Fatouros-Bergman, H., Flyckt, L., Ford, J.M., Fouche, J.-P., Fukunaga, M., Gill, M., Glahn, D.C., Gollub, R., Goudzwaard, E.D., Guo, H., Gur, R.E., Gur, R.C., Gurholt, T.P., Hashimoto, R., Hatton, S.N., Henskens, F.A., Hibar, D.P., Hickie, I.B., Hong, L.E., Horacek, J., Howells, F.M., Hulshoff Pol, H.E., Hyde, C.L., Isaev, D., Jablensky, A., Jansen, P.R., Janssen, J., Jönsson, E.G., Jung, L.A., Kahn, R.S., Kikinis, Z., Liu, K., Klauser, P., Knöchel, C., Kubicki, M., Lagopoulos, J., Langen, C., Lawrie, S., Lenroot, R.K., Lim, K.O., Lopez-Jaramillo, C., Lyall, A., Magnotta, V., Mandl, R.C.W., Mathalon, D.H., McCarley, R.W., McCarthy-Jones, S., McDonald, C., McEwen, S., McIntosh, A., Melicher, T., Mesholam-Gately, R.I., Michie, P.T., Mowry, B., Mueller, B.A., Newell, D.T., O’Donnell, P., Oertel-Knöchel, V., Oestreich, L., Paciga, S.A., Pantelis, C., Pasternak, O., Pearlson, G., Pellicano, G.R., Pereira, A., Pineda Zapata, J., Piras, F., Potkin, S.G., Preda, A., Rasser, P.E., Roalf, D.R., Roiz, R., Roos, A., Rotenberg, D., Satterthwaite, T.D., Savadjiev, P., Schall, U., Scott, R.J., Seal, M.L., Seidman, L.J., Shannon Weickert, C., Whelan, C.D., Shenton, M.E., Kwon, J.S., Spalletta, G., Spaniel, F., Sprooten, E., Stäblein, M., Stein, D.J., Sundram, S., Tan, Y., Tan, S., Tang, S., Temmingh, H.S., Westlye, L.T., Tønnesen, S., Tordesillas-Gutierrez, D., Doan, N.T., Vaidya, J., van Haren, N.E.M., Vargas, C.D., Vecchio, D., Velakoulis, D., Voineskos, A., Voyvodic, J.Q., Wang, Z., Wan, P., Wei, D., Weickert, T.W., Whalley, H., White, T., Whitford, T.J., Wojcik, J.D., Xiang, H., Xie, Z., Yamamori, H., Yang, F., Yao, N., Zhang, G., Zhao, J., van Erp, T.G.M., Turner, J., Thompson, P.M., Donohoe, G., 2018. Widespread white matter microstructural differences in schizophrenia across 4322 individuals: results from the ENIGMA Schizophrenia DTI Working Group. Mol. Psychiatry 23, 1261-1269. https://doi.org/10.1038/mp.2017.170

Khundrakpam, B.S., Tohka, J., Evans, A.C., 2015. Prediction of brain maturity based on cortical thickness at different spatial resolutions. NeuroImage 111, 350-359. https://doi.org/10.1016/j.neuroimage.2015.02.046

Kirkpatrick, B., Messias, E., Harvey, P.D., Fernandez-Egea, E., Bowie, C.R., 2008. Is Schizophrenia a Syndrome of Accelerated Aging? Schizophr. Bull. 34, 1024-1032. https://doi.org/10.1093/schbul/sbm140 
Knowles, E.E.M., David, A.S., Reichenberg, A., 2010. Processing speed deficits in schizophrenia: reexamining the evidence. Am. J. Psychiatry 167, 828-835. https://doi.org/10.1176/appi.ajp.2010.09070937

Kochunov, P., Chiappelli, J., Hong, L.E., 2013a. Permeability-diffusivity modeling vs. fractional anisotropy on white matter integrity assessment and application in schizophrenia. NeuroImage Clin. 3, 18-26. https://doi.org/10.1016/j.nicl.2013.06.019

Kochunov, P., Chiappelli, J., Wright, S.N., Rowland, L.M., Patel, B., Wijtenburg, S.A., Nugent, K., McMahon, R.P., Carpenter, W.T., Muellerklein, F., Sampath, H., Hong, L.E., 2014. Multimodal white matter imaging to investigate reduced fractional anisotropy and its age-related decline in schizophrenia. Psychiatry Res. 223, 148-156. https://doi.org/10.1016/j.pscychresns.2014.05.004

Kochunov, P., Coyle, T.R., Rowland, L.M., Jahanshad, N., Thompson, P.M., Kelly, S., Du, X., Sampath, H., Bruce, H., Chiappelli, J., Ryan, M., Fisseha, F., Savransky, A., Adhikari, B., Chen, S., Paciga, S.A., Whelan, C.D., Xie, Z., Hyde, C.L., Chen, X., Schubert, C.R., O’Donnell, P., Hong, L.E., 2017. Association of White Matter With Core Cognitive Deficits in Patients With Schizophrenia. JAMA Psychiatry 74, 958-966. https://doi.org/10.1001/jamapsychiatry.2017.2228

Kochunov, P., Ganjgahi, H., Winkler, A., Kelly, S., Shukla, D.K., Du, X., Jahanshad, N., Rowland, L., Sampath, H., Patel, B., O’Donnell, P., Xie, Z., Paciga, S.A., Schubert, C.R., Chen, J., Zhang, G., Thompson, P.M., Nichols, T.E., Hong, L.E., 2016a. Heterochronicity of white matter development and aging explains regional patient control differences in schizophrenia. Hum. Brain Mapp. 37, 4673-4688. https://doi.org/10.1002/hbm.23336

Kochunov, P., Glahn, D.C., Rowland, L.M., Olvera, R.L., Winkler, A., Yang, Y.-H., Sampath, H., Carpenter, W.T., Duggirala, R., Curran, J., Blangero, J., Hong, L.E., 2013b. Testing the hypothesis of accelerated cerebral white matter aging in schizophrenia and major depression. Biol. Psychiatry 73, 482-491. https://doi.org/10.1016/j.biopsych.2012.10.002

Kochunov, P., Hong, L.E., 2014. Neurodevelopmental and neurodegenerative models of schizophrenia: white matter at the center stage. Schizophr. Bull. 40, 721-728. https://doi.org/10.1093/schbul/sbu070

Kochunov, P., Huang, J., Chen, Song, Li, Y., Tan, S., Fan, F., Feng, W., Wang, Y., Rowland, L.M., Savransky, A., Du, X., Chiappelli, J., Chen, Shuo, Jahanshad, N., Thompson, P.M., Ryan, M.C., Adhikari, B., Sampath, H., Cui, Y., Wang, Z., Yang, F., Tan, Y., Hong, L.E., 2019. White Matter in Schizophrenia Treatment Resistance. Am. J. Psychiatry 176, 829-838. https://doi.org/10.1176/appi.ajp.2019.18101212

Kochunov, P., Rowland, L.M., Fieremans, E., Veraart, J., Jahanshad, N., Eskandar, G., Du, X., Muellerklein, F., Savransky, A., Shukla, D., Sampath, H., Thompson, P.M., Hong, L.E., 2016b. Diffusionweighted imaging uncovers likely sources of processing-speed deficits in schizophrenia. Proc. Natl. Acad. Sci. U. S. A. 113, 13504-13509. https://doi.org/10.1073/pnas.1608246113

Kochunov, P., Zavaliangos-Petropulu, A., Jahanshad, N., Thompson, P.M., Ryan, M.C., Chiappelli, J., Chen, S., Du, X., Hatch, K., Adhikari, B., Sampath, H., Hare, S., Kvarta, M., Goldwaser, E., Yang, F., Olvera, R.L., Fox, P.T., Curran, J.E., Blangero, J., Glahn, D.C., Tan, Y., Hong, L.E., 2020. A White Matter Connection of Schizophrenia and Alzheimer's Disease. Schizophr. Bull. https://doi.org/10.1093/schbul/sbaa078

Koutsouleris, N., Davatzikos, C., Borgwardt, S., Gaser, C., Bottlender, R., Frodl, T., Falkai, P., RiecherRössler, A., Möller, H.-J., Reiser, M., Pantelis, C., Meisenzahl, E., 2014. Accelerated Brain Aging in Schizophrenia and Beyond: A Neuroanatomical Marker of Psychiatric Disorders. Schizophr. Bull. 40, 1140-1153. https://doi.org/10.1093/schbul/sbt142

Liem, F., Varoquaux, G., Kynast, J., Beyer, F., Kharabian Masouleh, S., Huntenburg, J.M., Lampe, L., Rahim, M., Abraham, A., Craddock, R.C., Riedel-Heller, S., Luck, T., Loeffler, M., Schroeter, M.L., Witte, A.V., Villringer, A., Margulies, D.S., 2017. Predicting brain-age from multimodal imaging data captures cognitive impairment. NeuroImage 148, 179-188. https://doi.org/10.1016/j.neuroimage.2016.11.005 
Miyata, J., Yamada, M., Namiki, C., Hirao, K., Saze, T., Fujiwara, H., Shimizu, M., Kawada, R., Fukuyama, H., Sawamoto, N., Hayashi, T., Murai, T., 2010. Reduced white matter integrity as a neural correlate of social cognition deficits in schizophrenia. Schizophr. Res. 119, 232-239. https://doi.org/10.1016/j.schres.2009.12.038

Mori, T., Ohnishi, T., Hashimoto, R., Nemoto, K., Moriguchi, Y., Noguchi, H., Nakabayashi, T., Hori, H., Harada, S., Saitoh, O., Matsuda, H., Kunugi, H., 2007. Progressive changes of white matter integrity in schizophrenia revealed by diffusion tensor imaging. Psychiatry Res. 154, 133-145. https://doi.org/10.1016/j.pscychresns.2006.09.004

Nazeri, A., Chakravarty, M.M., Felsky, D., Lobaugh, N.J., Rajji, T.K., Mulsant, B.H., Voineskos, A.N., 2013. Alterations of Superficial White Matter in Schizophrenia and Relationship to Cognitive Performance. Neuropsychopharmacology 38, 1954-1962. https://doi.org/10.1038/npp.2013.93

Nenadić, I., Dietzek, M., Langbein, K., Sauer, H., Gaser, C., 2017. BrainAGE score indicates accelerated brain aging in schizophrenia, but not bipolar disorder. Psychiatry Res. Neuroimaging 266, 86-89. https://doi.org/10.1016/j.pscychresns.2017.05.006

Pérez-Iglesias, R., Tordesillas-Gutiérrez, D., McGuire, P.K., Barker, G.J., Roiz-Santiañez, R., Mata, I., de Lucas, E.M., Rodríguez-Sánchez, J.M., Ayesa-Arriola, R., Vazquez-Barquero, J.L., CrespoFacorro, B., 2010. White Matter Integrity and Cognitive Impairment in First-Episode Psychosis. Am. J. Psychiatry 167, 451-458. https://doi.org/10.1176/appi.ajp.2009.09050716

Ribe, A.R., Laursen, T.M., Charles, M., Katon, W., Fenger-Grøn, M., Davydow, D., Chwastiak, L., Cerimele, J.M., Vestergaard, M., 2015. Long-term Risk of Dementia in Persons With Schizophrenia: A Danish Population-Based Cohort Study. JAMA Psychiatry 72, 1095-1101. https://doi.org/10.1001/jamapsychiatry.2015.1546

Roalf, D.R., Ruparel, K., Verma, R., Elliott, M.A., Gur, R.E., Gur, R.C., 2013. White matter organization and neurocognitive performance variability in schizophrenia. Schizophr. Res. 143, 172-178. https://doi.org/10.1016/j.schres.2012.10.014

Shahab, S., Mulsant, B.H., Levesque, M.L., Calarco, N., Nazeri, A., Wheeler, A.L., Foussias, G., Rajji, T.K., Voineskos, A.N., 2019. Brain structure, cognition, and brain age in schizophrenia, bipolar disorder, and healthy controls. Neuropsychopharmacology 44, 898. https://doi.org/10.1038/s41386-018-0298-z

Smith, S.M., Vidaurre, D., Alfaro-Almagro, F., Nichols, T.E., Miller, K.L., 2019. Estimation of brain age delta from brain imaging. NeuroImage 200, 528-539. https://doi.org/10.1016/j.neuroimage.2019.06.017

Steffener, J., Habeck, C., O’Shea, D., Razlighi, Q., Bherer, L., Stern, Y., 2016. Differences between chronological and brain age are related to education and self-reported physical activity. Neurobiol. Aging 40, 138-144. https://doi.org/10.1016/j.neurobiolaging.2016.01.014

Voineskos, A.N., Felsky, D., Kovacevic, N., Tiwari, A.K., Zai, C., Chakravarty, M.M., Lobaugh, N.J., Shenton, M.E., Rajji, T.K., Miranda, D., Pollock, B.G., Mulsant, B.H., McIntosh, A.R., Kennedy, J.L., 2013. Oligodendrocyte Genes, White Matter Tract Integrity, and Cognition in Schizophrenia. Cereb. Cortex 23, 2044-2057. https://doi.org/10.1093/cercor/bhs188

Wang, J., Knol, M.J., Tiulpin, A., Dubost, F., Bruijne, M. de, Vernooij, M.W., Adams, H.H.H., Ikram, M.A., Niessen, W.J., Roshchupkin, G.V., 2019. Gray Matter Age Prediction as a Biomarker for Risk of Dementia. Proc. Natl. Acad. Sci. 116, 21213-21218. https://doi.org/10.1073/pnas.1902376116

Wang, R., Lagakos, S.W., Ware, J.H., Hunter, D.J., Drazen, J.M., 2007. Statistics in Medicine — Reporting of Subgroup Analyses in Clinical Trials. N. Engl. J. Med. 357, 2189-2194. https://doi.org/10.1056/NEJMsr077003

Wechsler, D., 1999. Wechsler Abbreviated Scale of Intelligence. New York, NY.: The Psychological Corporation.

Wood, S.N., 2011. Fast stable restricted maximum likelihood and marginal likelihood estimation of semiparametric generalized linear models. J. R. Stat. Soc. Ser. B Stat. Methodol. 73, 3-36. https://doi.org/10.1111/j.1467-9868.2010.00749.x 
1

2

3

4

5

6

7

8

9

Wright, S., Kochunov, P., Chiappelli, J., McMahon, R., Muellerklein, F., Wijtenburg, S.A., White, M.G., Rowland, L.M., Hong, L.E., 2014. Accelerated white matter aging in schizophrenia: role of white matter blood perfusion. Neurobiol. Aging 35, 2411-2418. https://doi.org/10.1016/j.neurobiolaging.2014.02.016

Zeng, B., Ardekani, B.A., Tang, Y., Zhang, T., Zhao, S., Cui, H., Fan, X., Zhuo, K., Li, C., Xu, Y., Goff, D.C., Wang, J., 2016. Abnormal white matter microstructure in drug-naive first episode schizophrenia patients before and after eight weeks of antipsychotic treatment. Schizophr. Res. 172, 1-8. https://doi.org/10.1016/j.schres.2016.01.051 